\title{
Cyber Psychology: A Qualitative study to deviate black hat hacker teenagers towards white hat hacker teenagers in Mauritius.
}

\begin{abstract}
Escalation of the internet use has brought many good things such as electronic commerce, easy access to vast store of reference material, emails and new avenues for advertising and so on. Technology is also placing teenagers in front of diverse choices that the virtual world enables. Along with technology advancement, there is also a dark side related to it: Black Hat Hacker. A black hat hacker is a person who attempts to find computer security vulnerabilities and exploit them for personal financial information, compromising the security of major systems or altering the functions of websites. Many Cyber Security researchers have enlightened studies on how can companies and governments prevent their systems from being hacked as well as what are the underlying purposes behind a black hat hacker's actions.

However, from a Cyber Psychological perspective, little empirical research has been conducted on teenagers showing black hat hacker's behavior. Hence, this research paper has focused mainly on the personalities of black hat hacker teenagers using Big 5 Personality Traits, Freud's theory explaining the reasons behind their actions, Motivation Theory behind their behavior towards black hat hacker, Self Control Theory and Interactional Theory of deviant behavior among teenagers who are computer users.

The main objective of this paper is to study why teenagers having great computer skills are more deviated towards black hat hacker. This is a qualitative research paper with 10 teenage participants aged from 11 years old to 18 years old from CyberStorm Members in Mauritius. These teenagers had been noticed with black hat hacker's mindset, behaviors and personality traits. But, under observation and guidance during 6 months, it was detected that their deviant behavior had been shifted from black hat hackers to white hat hackers. This empirical paper was also undertaken as there is an urgent need to develop effective prevention and intervention strategies for young people leading towards cybercrime. Additionally, there is a need to promote positive ways of channeling young talent toward careers in the tech sector. It is envisaged that the research findings will inform the work of professionals within key infrastructures under threat from particular types of cyber-criminality (i.e. fraud and financial crimes against industry), will contribute to educational awareness in the home, in schools and colleges particularly for vulnerable young people, as well as inform the development of training and best practice for policing across a range of roles and cyber-offences.
\end{abstract}


Author: Veediasha Bekaroo \& Loganaden Velvindron

Email Address: veediasha.bekaroo@gmail.com; loganaden@gmail.com

Keywords: Cyber Psychology, Technology, cybercrime, CyberStorm Members, Black hat hacker teenager, deviant behavior. 